\title{
Orientation factors in three-centre energy pooling
}

\author{
Robert D. Jenkins and David L. Andrews* \\ School of Chemical Sciences, University of East Anglia, Norwich, UK NR4 7TJ. \\ E-mail: robert.jenkins@uea.ac.uk and d.l.andrews@uea.ac.uk
}

\author{
Received 22nd March 2000, Accepted 9th May 2000 \\ Published on the Web 12th June 2000
}

\begin{abstract}
At high levels of optical illumination, multichromophore arrays can exhibit a novel form of resonance energy transfer involving the coupling of two donors and one acceptor chromophore. This three-centre energy pooling is the subject of an investigation into its dependence on the mutual positions and orientations of the participating chromophores. Employing results obtained through quantum electrodynamical calculation, comparisons are made between pairwise resonance energy transfer and the novel three-body analogue, with counterparts to the conventional orientation factor identified. The interplay of such factors is analysed in the context of molecular architectures based on linear configurations. The relative dominance of cooperative and accretive mechanisms for energy pooling is addressed and the relative alignments and magnitudes of the donor and acceptor transition moments and polarisabilities are shown to have a profound effect on achievable pooling efficiency.
\end{abstract}

\section{Introduction}

The resonant transfer of energy from sites of excitation to other amenable sites, beyond wavefunction overlap, is a wellknown aspect of photophysics. Much of the interest in resonance energy transfer (RET) concerns systems where excitation transfers between donor and acceptor chromophores with a relatively fixed mutual orientation within a molecular assembly. Such processes play an essential role in a host of complex media, for example light migration within photosynthetic systems, ${ }^{1-3}$ intra- and interlayer energy exchange in Langmuir-Blodgett films, ${ }^{4,5}$ and, as recently observed, in self-assembled conjugated polymer superlattices. ${ }^{6}$ It also has an emergent role in vibrational energy redistribution in water, ${ }^{7}$ and in proteins it is increasingly employed as a means of determining internal distances, through measurement of ensuing fluorescence (FRET) ${ }^{8,9}$ Alongside these experimental observations, recent theory shows that RET can be significantly enhanced by an off-resonant laser beam (laser assisted resonance energy transfer). ${ }^{10,11}$

The basic phenomenon of energy transfer was, until recently, thought to proceed by one of two mechanisms: short-range radiationless transfer with an inverse sixth power distance dependence $^{12}$ or conversely a long-range radiative emissioncapture process following the familiar inverse square law. ${ }^{13}$ These two mechanisms have subsequently been identified as asymptotes of a unified theory operative over all post-overlap distances. ${ }^{14}$ This theory, whose classical precursor involved the incorporation of retardation features in dipole coupling, ${ }^{15}$ in its modern quantum electrodynamical form establishes the detailed distance-dependent interplay of retardation and quantum uncertainty. ${ }^{16}$

A great deal of attention has focused on the role of the structural link between donor and acceptor, commonly termed the bridge or spacer. ${ }^{17}$ Much research has revolved around the determination of interchromophore distances, both in systems where the bridges are rigid ${ }^{18,19}$ and in others where they are flexible, ${ }^{20,21}$ tasks well-suited to the data that RET studies produce. ${ }^{12}$ Furthermore the effects of spacer length, ${ }^{22}$ the nature of like and unlike chromophores, ${ }^{23}$ and orientation factors ${ }^{20,24}$ have been investigated. Recently the discussion has widened to multichromophore arrays, an area in which much synthetic effort is directed towards the production of efficient mimics of the photosynthetic light harvesting centre. ${ }^{25,26}$ In this context, the nature and evolution of single excitations within large molecular arrays has been elucidated for a variety of systems including cyclodextrins, ${ }^{27}$ multiporphyrins, ${ }^{28,29}$ and dendrimers. ${ }^{30,31}$

In all the commonly studied situations cited above, each and every fundamental energy transfer step takes place between one donor and one acceptor. The theory behind this pairwise (or bimolecular) energy migration, in systems accountable to post-overlap distance regimes, is welldocumented in the literature ${ }^{32,33}$ and serves simple situations well. However, recent work has revealed that bridging species may play a far less passive role, significantly modifying the transfer process. ${ }^{34}$ In such cases, it is no longer adequate to model the process as simply a donor/acceptor interaction, as the electronic influence of the bridging species has to be properly accounted for. ${ }^{35-38}$

At the high levels of laser illumination associated with the onset of optical nonlinearity, two or more electronic excitations are likely to coexist locally within a multichromophore array. If decay lifetimes are sufficiently long, such a situation will arise even at comparatively modest intensities. Now consider that also present in the array are chromophores whose energy levels are amenable to acquisition of the dual excitations via a novel energy transfer process. This has been termed three-body energy pooling. ${ }^{38-40}$ Here, and in the following, 'body' or 'centre' is a generic term for the donors and acceptors - either distinct molecules or chromophores. Such (non-collisional) pooling interactions can be described in simple chemical terms by the equation

$$
\mathrm{A}^{*}+\mathrm{B}+\mathrm{A}^{* *} \rightarrow \mathrm{A}+\mathrm{B}^{*}+\mathrm{A}^{\prime}
$$

with $\mathrm{A}$ and $\mathrm{A}^{\prime}$ being chemically identical donor species (the prime serving only to distinguish between them), B is the acceptor, and the asterisk indicates electronic excitation. It is important to note that the total energy transferred to the 


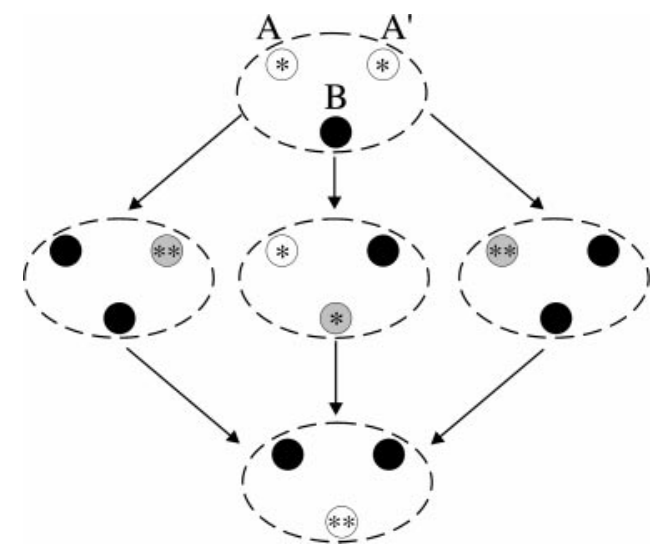

Fig. 1 Cartoon of different pooling pathways. On the left and right are accretive pathways, with energy accrued at $\mathrm{A}^{\prime}$ and A respectively; the central path shows cooperative pooling. White, black and shaded circles indicate excited, ground and virtual state species respectively, and asterisks represent the locale of excitation(s).

acceptor is less than or equal to the sum of initial excitations of both donors, any losses being due to initial vibronic relaxation of the donor excited states. Also it is to be understood that neither A nor $\mathrm{A}^{\prime}$ has an upper level suitable to accommodate the combined energy of two $\mathrm{A}^{*}$ states. Dual excitation delivery to a single acceptor site is not unlike two-photon FRET - the fundamental difference between the two is that, in the latter, a single energy transfer step is responsible for deposition of the two-photon excitation energy of a single donor. ${ }^{41,42}$

In various chemical disciplines, examples of this energy pooling are evident. ${ }^{43-45}$ Interestingly the architectural and photophysical properties of many supermolecular dendrimers and multichromophore arrays lend themselves to the exhibition of this novel process. Previously the authors have elucidated the existence and interplay of possible mechanistic pathways for three-body energy pooling, termed cooperative ${ }^{38}$ and accretive, ${ }^{40}$ as illustrated in Fig. 1. Specifically, the cooperative mechanism is concerned with direct pooling of donor excitation at the acceptor; accretive channelling is specifically where the excitation of one donor is fleetingly accrued by the second before net transfer to the acceptor. In pooling systems the prevalence of the cooperative and accretive mechanisms has been proven to show reverence to limited architectural features. ${ }^{39}$

\section{Theory}

In this section a concise summary of the theory leading to the key results is laid out. The reader keen to focus directly on the orientational features of those results is invited to skip ahead to Section 3.

As a fully fledged quantum theory of light and matter, molecular quantum electrodynamics (QED) is the proper platform on which to base investigations into three-body pooling processes. ${ }^{46}$ In terms of QED, conventional resonance energy transfer is described as a virtual photon exchange between a pre-excited donor and a ground state acceptor. The messenger photon is deemed virtual due to its unobservable nature, and its correct representation invokes a sum over all possible wave-vectors and polarisations. To account fully for any intervening medium effects, descriptions in terms of photons (which strictly represent vacuum energy propagation) are here superseded by a formulation in terms of polaritons. ${ }^{47}$

Consider the three-body energy pooling system comprising two chemically identical donors $\mathrm{A}$ and $\mathrm{A}^{\prime}$ along with a chemi- cally independent acceptor, B. The Hamiltonian for such a system can be written as

$$
H=H_{\text {bath }}+\sum_{\xi=\mathbf{A}, \mathbf{A}^{\prime}, \mathbf{B}} H_{\mathrm{mol}}^{\xi}+\sum_{\xi=\mathbf{A}, \mathbf{A}^{\prime}, \mathbf{B}} H_{\mathrm{int}}^{\xi} .
$$

In eqn. (2.1) the polariton bath Hamiltonian is explicitly

$$
H_{\text {bath }}=H_{\text {rad }}+\sum_{\xi \neq \mathrm{A}, \mathrm{A}^{\prime}, \mathrm{B}}\left(H_{\mathrm{mol}}^{\xi}+H_{\mathrm{int}}^{\xi}\right)
$$

in which $H_{\mathrm{rad}}$ is the second-quantised radiation field Hamiltonian. In eqns. (2.1) and (2.2), $H_{\mathrm{mol}}^{\xi}$ and $H_{\mathrm{mt}}^{\xi}$ are the molecular and molecule-field coupling Hamiltonians respectively for molecule $\xi$. At distances beyond wavefunction overlap where the electric dipole approximation can be implemented, the latter operator is defined by

$$
H_{\text {int }}^{\xi}=-\varepsilon_{0}^{-1} \boldsymbol{\mu}(\xi) \cdot d^{\perp}\left(\boldsymbol{R}_{\xi}\right)
$$

with $\boldsymbol{\mu}(\xi)$ being the electric dipole moment operator and $\boldsymbol{R}_{\xi}$ the position vector for $\xi$. As discussed elsewhere, ${ }^{48,49}$ the electric displacement field operator, $\boldsymbol{d}^{\perp}\left(R_{\xi}\right)$, may be cast in terms of a mode expansion modified to accommodate the influence of the intervening medium. Explicitly, this expansion for the polariton-mediated exchange is given by

$$
\begin{aligned}
\boldsymbol{d}^{\perp}\left(\boldsymbol{R}_{\xi}\right)= & \mathrm{i} \sum_{\boldsymbol{p}} \sum_{m} \sum_{\lambda=1}^{2}\left(\frac{\varepsilon_{0} \hbar \omega_{p}^{(m)} v_{\mathbf{g}}^{(m)}}{2 c V_{0} n^{(m)}}\right)^{1 / 2}\left(\frac{\left(n^{(m)}\right)^{2}+2}{3}\right) \\
& \times\left[\boldsymbol{e}^{(\lambda)}(\boldsymbol{p}) P_{\boldsymbol{p}, m, \lambda} \mathrm{e}^{\mathrm{i} \boldsymbol{p} \cdot \boldsymbol{R}_{\xi}}-\overline{\boldsymbol{e}}^{(\lambda)}(\boldsymbol{p}) P_{\boldsymbol{p}, m, \lambda}^{\dagger} \mathrm{e}^{-\mathrm{i} \boldsymbol{p} \cdot \boldsymbol{R}_{\xi}}\right] .
\end{aligned}
$$

Expression (2.4) embraces a summation over polariton modes characterised by wave-vector $\boldsymbol{p}$, polarisation $\lambda$ [unit vector $\boldsymbol{e}^{(\lambda)}(\boldsymbol{p})$ and complex conjugate $\overline{\boldsymbol{e}}^{(\lambda)}(\boldsymbol{p}]$, and frequency $\omega_{p}^{(m)}$. The index $m$ is included to indicate branches of polariton dispersion over which the refractive index is $n^{(m)}$ and the group velocity $v_{\mathrm{g}}^{(m)}$. Moreover $P$ and $P^{\dagger}$ are the polariton annihilation and creation operators respectively and $V_{0}$ is the quantisation volume.

The overall rate, $\Gamma$, for energy transfer processes is expressible via Fermi's golden rule

$$
\Gamma=\frac{2 \pi}{\hbar}\left|M_{f i}\right|^{2} \rho_{\beta}
$$

where $\rho_{\beta}$ is the density of final states of the acceptor dependent on its internal vibronic structure. The probability amplitude or matrix element, $M_{f i}$, for three-centre energy pooling is described by the fourth-order term in the time-dependent perturbation expansion

$$
M_{f i}=\sum_{r, s, t} \frac{\left\langle f\left|H_{\mathrm{int}}\right| t\right\rangle\left\langle t\left|H_{\mathrm{int}}\right| s\right\rangle\left\langle s\left|H_{\mathrm{int}}\right| r\right\rangle\left\langle r\left|H_{\mathrm{int}}\right| i\right\rangle}{\left(E_{i}-E_{r}\right)\left(E_{i}-E_{s}\right)\left(E_{i}-E_{t}\right)}
$$

where initial and final system states, $|i\rangle$ and $|f\rangle$, are coupled through virtual states $|r\rangle,|s\rangle$ and $|t\rangle$. In general, each of the system states, $|n\rangle$, featuring in (2.6) has energy $E_{n}$ and takes the form of a product of the eigenstates of $H_{\mathrm{mol}}^{\mathrm{A}}, H_{\mathrm{mol}}^{\mathrm{A}^{\prime}}, H_{\mathrm{mol}}^{\mathrm{B}}$ and $H_{\text {bath }}$. The latter Hamiltonian differs from $H_{\text {rad }}$ through explicit accommodation of the host medium interaction, so that its quanta are correctly described as polaritons.

By following techniques detailed elsewhere, ${ }^{16,38-40}$ effecting the summation of all contributions to (2.6) leads to the identification of three groups of terms corresponding to physically discernible features of the pooling process, and characterised as follows:

$$
M_{f i}=M_{f i}^{\mathrm{coop}}+M_{f i}^{\mathrm{accl}}+M_{f i}^{\mathrm{acc} 2}
$$

(see Fig. 1). The right-hand side of (2.7) comprises contributions associated with the cooperative mechanism $\left(M_{f i}^{\text {coop }}\right)$ along with two accretive terms associated with energy being accrued 
at either A $\left(M_{f i}^{\mathrm{accl}}\right)$ or $\mathrm{A}^{\prime}\left(M_{f i}^{\mathrm{acc} 2}\right)$. Explicitly, the three individual contributions to eqn. (2.7) may be written as

$$
\begin{aligned}
& M_{f i}^{\text {coop }}=\mu_{i}^{0 \alpha(\mathrm{A})} V_{i j}(n k, \boldsymbol{R}) \alpha_{j k}^{\beta 0(\mathrm{~B})}(-k,-k) V_{k l}\left(n k, \boldsymbol{R}^{\prime}\right) \mu_{l}^{0 \alpha\left(\mathrm{A}^{\prime}\right)} \\
& M_{f i}^{\mathrm{accl}}=\mu_{i}^{0 \alpha(\mathrm{A})} V_{i j}\left(n k, \boldsymbol{R}^{\prime \prime}\right) \alpha_{j k}^{\beta 0\left(\mathrm{~A}^{\prime}\right)}(2 k,-k) V_{k l}\left(2 n k, \boldsymbol{R}^{\prime}\right) \mu_{l}^{0 \alpha(\mathbf{B})}
\end{aligned}
$$

and

$$
M_{f i}^{\mathrm{acc} 2}=\mu_{i}^{0 \alpha\left(\mathbf{A}^{\prime}\right)} V_{i j}\left(n k, \boldsymbol{R}^{\prime \prime}\right) \alpha_{j k}^{\beta 0(\mathbf{A})}(2 k,-k) V_{k l}(2 n k, \boldsymbol{R}) \mu_{l}^{0 \alpha(\mathbf{B})}
$$

introducing transition dipole moments $\boldsymbol{\mu}^{x y(\xi)}=\left\langle x\left|\boldsymbol{\mu}^{(\xi)}\right| y\right\rangle$, intermolecular separation vectors $\boldsymbol{R}=\boldsymbol{R}_{\mathrm{B}}-\boldsymbol{R}_{\mathrm{A}}, \quad \boldsymbol{R}^{\prime}=\boldsymbol{R}_{\mathrm{B}}$ $-\boldsymbol{R}_{\mathrm{A}^{\prime}}$ and $\boldsymbol{R}^{\prime \prime}=\boldsymbol{R}_{\mathrm{A}^{\prime}}-\boldsymbol{R}_{\mathrm{A}}$, and implementing the rule of summation over repeated Cartesian indices. Eqns. (2.8) also introduce the energy identities $E_{\alpha}^{\mathrm{A}}-E_{0}^{\mathrm{A}}=E_{\alpha}^{\mathrm{A}^{\prime}}-E_{0}^{\mathrm{A}^{\prime}} \equiv E_{\alpha 0}^{\mathrm{A}}$ and $2 E_{\alpha 0}^{\mathrm{A}}=E_{\beta 0}^{\mathrm{B}}=2 \hbar c k$, signifying that the chemically equivalent $\mathrm{A}$ and $\mathrm{A}^{\prime}$ have an initial excitation energy of $\hbar c k$. For the reasons stated in Section 1 however, $\hbar c k$ is not to be identified with the photon energy which initiates the optical excitation, since non-radiative losses may be incurred.

The form and behaviour of the two types of second-rank tensor $\boldsymbol{\alpha}^{f i(\xi)}\left(k_{1}, k_{2}\right)$ and $\boldsymbol{V}(n K, \boldsymbol{r})$ featured in eqns. (2.8) invite further discussion and development. The two-photon interaction tensor, $\boldsymbol{\alpha}^{f i(\xi)}\left(k_{1}, k_{2}\right)$, has the general form

$$
\alpha_{i j}^{f i(\xi)}\left(\mp k_{1}, \mp k_{2}\right)=\sum_{\zeta}\left\{\frac{\mu_{i}^{f \zeta(\xi)} \mu_{j}^{\zeta i(\xi)}}{\left(\widetilde{E}_{i \zeta} \pm \hbar c k_{1}\right)}+\frac{\mu_{j}^{f \zeta(\xi)} \mu_{i}^{i(\xi)}}{\left(\widetilde{E}_{i \zeta} \pm \hbar c k_{2}\right)}\right\} .
$$

Eqn. (2.9) invokes a resonance modification to the energy denominators, associated with the virtual state $\zeta$ through which the molecule $\xi$ progresses. This is explicitly expressible as $\tilde{E}_{i \zeta}^{\xi}=E_{i \zeta}^{\xi}+\mathrm{i} \Gamma_{\zeta}$, where $\Gamma_{\zeta}$ represents the damping energy of the virtual state, the sign consistent with time-reversal symmetry. ${ }^{50,51}$ The form of the two-photon interaction tensor exhibits differing properties for alternative pooling pathways. For accretive mechanisms it plays the role of a variant on the well-known non-symmetric tensor identifiable in Raman scattering. ${ }^{52}$ For the cooperative mechanism the two-photon tensor exhibits behaviour similar to the index-symmetric twophoton absorption tensor. ${ }^{46}$

The results of eqns. (2.8) also contain the second-rank, index-symmetric, Cartesian, retarded resonance electric dipole-electric dipole coupling tensor, $\boldsymbol{V}(n K, \boldsymbol{r})$. Its general form, which again fully accommodates effects of the intervening transfer medium, is given by

$$
\begin{aligned}
V_{i j}(n K, \boldsymbol{r})= & \frac{1}{n^{2}}\left(\frac{n^{2}+2}{3}\right)^{2} \frac{\mathrm{e}^{\mathrm{i} n K r}}{4 \pi \varepsilon_{0} r^{3}}\{(1-\mathrm{i} n K r) \\
& \left.\times\left(\delta_{i j}-3 \hat{r}_{i} \hat{r}_{j}\right)-n^{2} K^{2} r^{2}\left(\delta_{i j}-\hat{r}_{i} \hat{r}_{j}\right)\right\} .
\end{aligned}
$$

Here, in the arguments on the left, $n$ is the refractive index appropriate for $K$, which relates to the magnitude of the energy transferral, and $r$ is a general distance between the connected transition electric dipoles. The behaviour of eqn. (2.10) necessarily approaches that of the vacuum case when the refractive index of the medium (and hence the Lorentz premultiplier) approaches unity. It is within the bounds of eqn. (2.10) that the power of the unified theory is apparent, as the short-range $(n K r \gg 1)$ and long-range $(n K r \ll 1)$ limits revealing $r^{-3}$ and $r^{-1}$ distance dependence in $\boldsymbol{V}(n K, \boldsymbol{r})$ respectively. ${ }^{14}$ Hereafter, interest primarily lies with the short-range asymptote, explicitly given by

$$
V_{i j}(0, r)=\frac{1}{n^{2}}\left(\frac{n^{2}+2}{3}\right)^{2} \frac{\left(\delta_{i j}-3 \hat{r}_{i} \hat{r}_{j}\right)}{4 \pi \varepsilon_{0} r^{3}}
$$

analogous to the instantaneous coupling between two electric dipoles in a molecular medium. ${ }^{46}$

\section{Orientational factors}

A well-known feature of the rate of pairwise resonance energy transfer is its dependence on the mutual orientation between molecular transition dipole moments, and also their orientation with respect to the intermolecular vector, as embodied in the traditional orientation factor $\kappa \cdot{ }^{12,53-55}$ This parameter takes values between 0 and 4 and is explicitly given by

$$
\kappa^{2}=(\cos \theta-3 \cos \phi \cos \gamma)^{2}
$$

where $\theta$ is the angle between the two transition moments, $\phi$ is the angle between the donor transition and the intermolecular vector, and $\gamma$ is the angle between the acceptor transition and the intermolecular vector. In the rapid diffusion or total disorder limit the mean value of the orientation factor $\left\langle\kappa^{2}\right\rangle=\frac{2}{3}$, a value often sufficiently adequate for use in the first interpretation of FRET measurements ${ }^{9,54,56}$ - although other values, taking into account statistical weighting factors, have been employed. ${ }^{57}$ Systems with more complicated pair architecture have also been analysed leading, not surprisingly, to substantially more complicated averaged expressions even in the rapid diffusion limit. ${ }^{58}$ There is much intrinsic interest in the various mathematical attributes of $\kappa$ and much discussion surrounding it. ${ }^{53-59}$

A simple two-centre energy transfer system requires only three angles to describe the nature of the orientation factors, as in eqn. (3.1). ${ }^{54}$ The addition of a non-passive third body, as in three-body energy pooling, considerably complicates matters. Consider a molecular triad representable by the coordinate system shown in Fig. 2, where the acceptor chromophore is situated at the origin, one donor necessarily lies on the chosen $x$-axis and the whole three-centre system lies within the $x y$-plane. To begin it is useful to view the system as being fully represented by five groups of three angles namely

$$
\begin{gathered}
\phi_{\xi} \text {-the angle between } \boldsymbol{\mu}^{\xi} \text { and } \hat{\boldsymbol{R}} \\
\theta_{\xi} \text {-the angle between } \boldsymbol{\mu}^{\xi} \text { and } \hat{\boldsymbol{R}}^{\prime} \\
\gamma_{\xi} \text {-the angle between } \boldsymbol{\mu}^{\xi} \text { and } \hat{\boldsymbol{R}}^{\prime \prime} \\
\Phi_{\boldsymbol{r} \boldsymbol{r}^{\prime}} \text {-the angle between } \hat{\boldsymbol{r}} \text { and } \hat{\boldsymbol{r}}^{\prime} \\
\Theta_{\xi \xi^{\prime}} \text {-the angle between } \boldsymbol{\mu}^{\xi} \text { and } \boldsymbol{\mu}^{\xi^{\prime}}
\end{gathered}
$$

with $\xi$ representing chromophores $\mathrm{A}, \mathrm{A}^{\prime}$ or $\mathrm{B}$. The 15 angles may be expressed in terms of a reduced, independent set of 12 variables (the number of internal degrees of freedom in this six-vector system) by trigonometric means. Application of the cosine rule yields

$$
\cos \Phi_{R R^{\prime \prime}}=\frac{R-R^{\prime} \cos \Phi}{\left(R^{2}+R^{\prime 2}-2 R R^{\prime} \cos \Phi\right)^{1 / 2}}
$$

and

$$
\cos \Phi_{R^{\prime} R^{\prime \prime}}=\frac{R^{\prime}-R \cos \Phi}{\left(R^{2}+R^{\prime 2}-2 R R^{\prime} \cos \Phi\right)^{1 / 2}}
$$

allowing release of the parameters $R^{\prime \prime}, \Phi_{R R^{\prime \prime}}$ and $\Phi_{R^{\prime} R^{\prime \prime}}$ in favour of $\Phi_{R R^{\prime}} \equiv \Phi, \boldsymbol{R}$ and $\boldsymbol{R}^{\prime}$. Use of the identity

$$
\boldsymbol{R}^{\prime \prime}=R \hat{\boldsymbol{R}}-R^{\prime} \hat{\boldsymbol{R}}^{\prime}
$$

then leads to the relationship

$$
\cos \gamma_{\xi}=\frac{R \cos \phi_{\xi}-R^{\prime} \cos \theta_{\xi}}{\left(R^{2}+R^{\prime 2}-2 R R^{\prime} \cos \Phi\right)^{1 / 2}}
$$

The reduced set of variables for the following calculations is then as shown in Fig. 3.

The probability amplitudes of eqns. (2.8) invoke tensor contractions of the general polarisability tensor (2.9) with the 


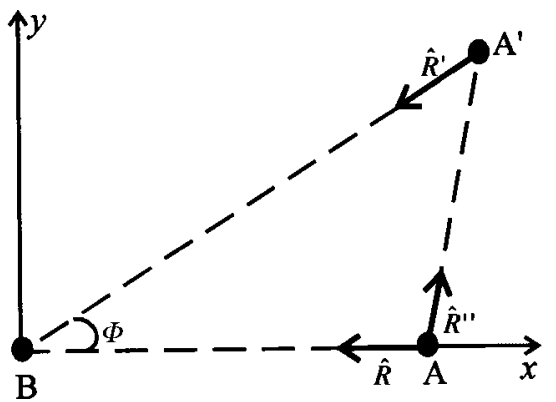

Fig. 2 Generalised co-ordinates for a three-centre system in the $x y$ plane.

short-range limit of $\boldsymbol{V}(n K, \boldsymbol{r})$, eqn. (2.11). The results are as follows:

$$
\begin{aligned}
M_{f i}^{\text {coop }} & =\frac{1}{n^{4}}\left(\frac{n^{2}+2}{3}\right)^{4} \frac{\left|\boldsymbol{\mu}^{\mathrm{A}}\left\|\boldsymbol{\mu}^{\mathrm{A}^{\prime}}\right\| \boldsymbol{\alpha}^{\mathrm{B}}\right| \kappa_{\mathrm{AB}} \kappa_{\mathrm{A}^{\prime} \mathrm{B}}}{\left(4 \pi \varepsilon_{0} R^{3}\right)\left(4 \pi \varepsilon_{0} R^{\prime 3}\right)} \\
M_{f i}^{\mathrm{acc} 1} & =\frac{1}{n^{4}}\left(\frac{n^{2}+2}{3}\right)^{4} \frac{\left|\boldsymbol{\mu}^{\mathrm{A}}\left\|\boldsymbol{\mu}^{\mathrm{B}}\right\| \boldsymbol{\alpha}^{\mathrm{A}^{\prime}}\right| \kappa_{\mathrm{A}^{\prime} \mathrm{B}} \kappa_{\mathrm{AA}^{\prime}}}{\left(4 \pi \varepsilon_{0} R^{\prime 3}\right)\left(4 \pi \varepsilon_{0} R^{\prime \prime 3}\right)}
\end{aligned}
$$

and

$$
M_{f i}^{\mathrm{acc} 2}=\frac{1}{n^{4}}\left(\frac{n^{2}+2}{3}\right)^{4} \frac{\left|\boldsymbol{\mu}^{\mathrm{A}^{\prime}}\left\|\boldsymbol{\mu}^{\mathrm{B}}\right\| \boldsymbol{\alpha}^{\mathrm{A}}\right| \kappa_{\mathrm{AB}} \kappa_{\mathrm{AA}}}{\left(4 \pi \varepsilon_{0} R^{3}\right)\left(4 \pi \varepsilon_{0} R^{\prime \prime 3}\right)} .
$$

Eqns. (3.6) contain new, kappa-like, parameters explicitly given by

$$
\begin{aligned}
\kappa_{\mathrm{AB}} & =\cos \Theta_{\mathrm{AB}}-3 \cos \phi_{\mathrm{A}} \cos \phi_{\mathrm{B}} \\
\kappa_{\mathrm{A}^{\prime} \mathrm{B}} & =\cos \Theta_{\mathrm{A}^{\prime} \mathrm{B}}-3 \cos \theta_{\mathrm{A}^{\prime}} \cos \theta_{\mathrm{B}} \\
\kappa_{\mathrm{AA}^{\prime}} & =\cos \Theta_{\mathrm{AA}^{\prime}}-3 \cos \gamma_{\mathrm{A}} \cos \gamma_{\mathrm{A}^{\prime}} .
\end{aligned}
$$

In deriving eqns. (3.6) the assumption is made for presentational simplicity that all transition moments (both real and virtual) associated with an individual molecule are collinear. For example transition moments associated with molecule A, real [as embraced by the polarisability tensor of $(2.8 \mathrm{a})$ ] or virtual [as in eqns. $(2.8 \mathrm{~b})$ and $(2.8 \mathrm{c})$ ], subtend a common angle $\phi_{\mathrm{A}}$ with the intermolecular unit vector $\hat{\boldsymbol{R}}$.

The system has been fully described above in terms of a minimum number of angles to allow adequate generality. To exemplify and illustrate the extraction of meaningful results

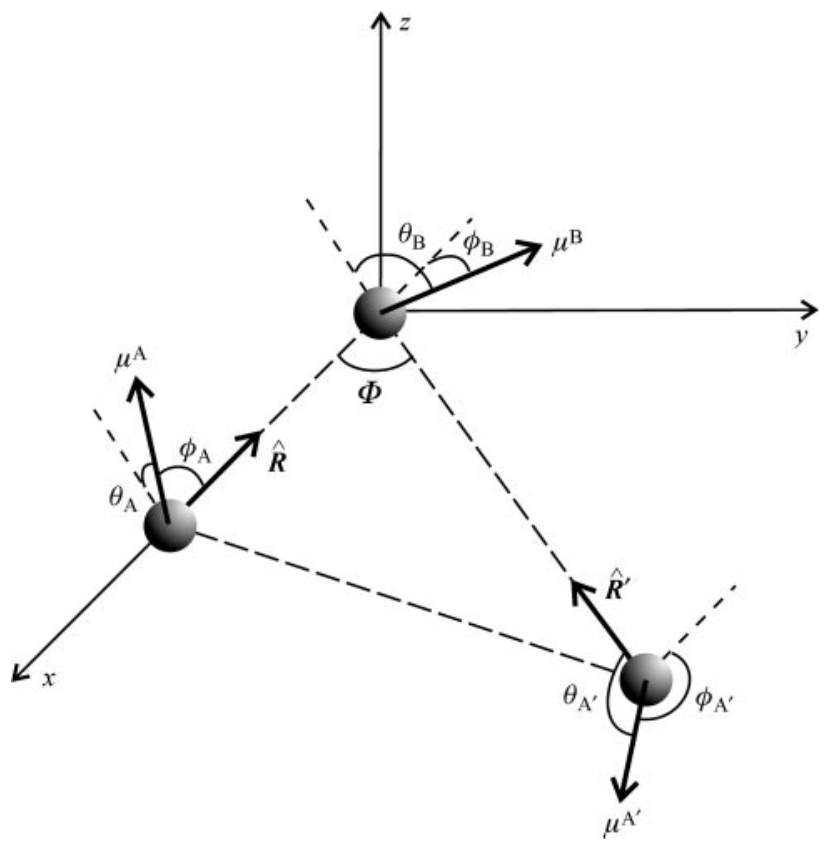

Fig. 3 Degrees of freedom for the internal geometry of a trichromophore array. from the given expressions it is expedient to reduce the number of variables. Here, more detailed investigations now focus on two related molecular geometries under physically realistic constraints from which graphable results can be obtained. Consider a linear molecular assembly, amenable to three-body energy transfer. The participant chromophores can adopt two fundamentally different arrangements - namely $\mathrm{AA}^{\prime} \mathrm{B}$ (equivalent to $\mathrm{A}^{\prime} \mathrm{AB}$ ) and $\mathrm{ABA}^{\prime}$ (equivalent to $\mathrm{A}^{\prime} \mathrm{BA}$ ). By inspection the first of these cases should give preference to the accretive mechanism and the second, conversely, should favour the cooperative pathway. Making the transition moments at both donors equal invokes $\Theta_{\mathrm{AA}^{\prime}}=0, \Theta_{\mathrm{AB}} \equiv$ $\Theta_{\mathrm{A}^{\prime} \mathrm{B}} \equiv \Theta$ and $\phi_{\mathrm{A}}=\phi_{\mathrm{A}^{\prime}}$, along with the ramifications shown in Table 1. Fig. 4 illustrates the molecular architecture for both $\mathrm{AA}^{\prime} \mathrm{B}$ and $\mathrm{ABA}^{\prime}$. Proper accommodation of these conditions in eqn. (2.7) leads to the following expressions for full quantum amplitude for three-body energy transfer, for the $\mathrm{ABA}^{\prime}$ and $\mathrm{AA}^{\prime} \mathrm{B}$ configurations:

$$
M_{f i}^{\mathrm{ABA}^{\prime}}=\frac{1}{n^{4}}\left(\frac{n^{2}+2}{3}\right)^{4} \frac{\left(C^{\mathrm{coop}}+\frac{1}{4} C^{\mathrm{acc}}\right)}{\left(4 \pi \varepsilon_{0} R^{3}\right)^{2}}
$$

and

$$
M_{f i}^{\mathrm{AA}{ }^{\prime} \mathrm{B}}=\frac{8}{n^{4}}\left(\frac{n^{2}+2}{3}\right)^{4} \frac{\left(C^{\mathrm{coop}}+9 C^{\mathrm{acc}}\right)}{\left(4 \pi \varepsilon_{0} R^{3}\right)^{2}} .
$$

It should be noted that each of the results (3.8) and (3.9) encompasses, with clearly different significance, contributions
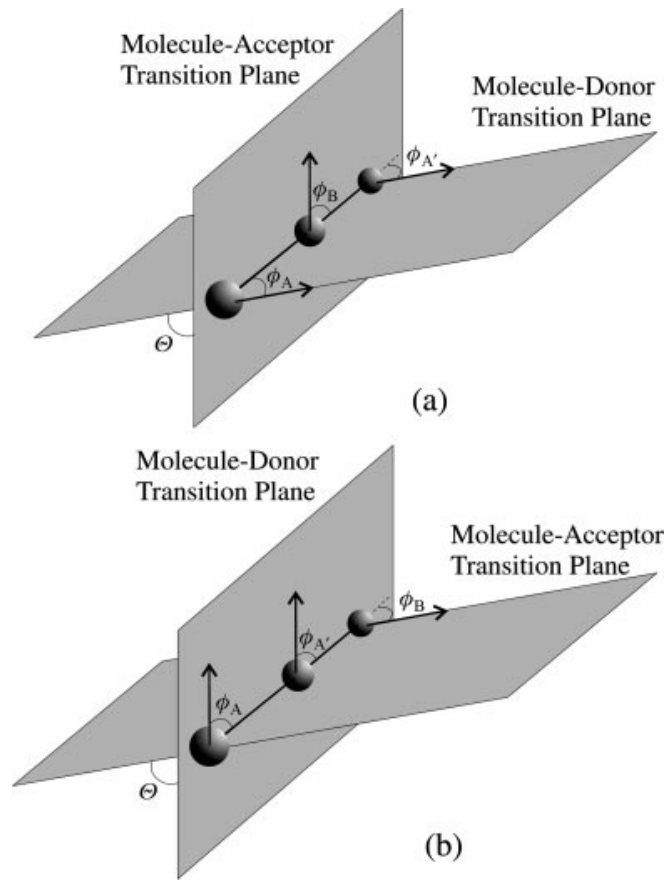

Fig. 4 Configurations for molecular triads (a) $\mathrm{ABA}^{\prime}$ and (b) $\mathrm{AA}^{\prime} \mathrm{B}$ explicitly showing angles $\phi_{\mathrm{A}}$ (between the transition moments at both $\mathrm{A}$ and $\mathrm{A}^{\prime}$ and the intermolecular vector $\hat{\boldsymbol{R}}$ ), $\phi_{\mathrm{B}}$ (between the transition moment at B and $\hat{\boldsymbol{R}}$ ), and $\Theta$ (between molecule-acceptor transition and molecule-donor transition planes).

Table 1 The pertinent quantities for chromophore arrays $\mathrm{ABA}^{\prime}$ and $\mathrm{AA}^{\prime} \mathrm{B}$

\begin{tabular}{lll}
\hline & $\mathrm{ABA}^{\prime}$ & $\mathrm{AA}^{\prime} \mathrm{B}$ \\
\hline$\Phi$ & $\pi$ & 0 \\
$R^{\prime}$ & $R$ & $R / 2$ \\
$R^{\prime \prime}$ & $2 R$ & $R / 2$ \\
$\cos \theta_{\xi}$ & $-\cos \phi_{\xi}$ & $\cos \phi_{\xi}$ \\
$\cos \gamma_{\xi}$ & $\cos \phi_{\xi}$ & $\cos \phi_{\xi}$ \\
\hline
\end{tabular}


from the cooperative and also both accretive mechanisms. These contributions are explicitly given by

$$
C^{\text {coop }}=\left|\boldsymbol{\mu}^{\mathrm{A}}\right|^{2}\left|\boldsymbol{\alpha}^{\mathrm{B}}\right|\left(\cos \Theta-3 \cos \phi_{\mathrm{A}} \cos \phi_{\mathrm{B}}\right)^{2}
$$

and

$$
C^{\mathrm{acc}}=\left|\boldsymbol{\mu}^{\mathrm{B}}\left\|\boldsymbol{\mu}^{\mathrm{A}}\right\| \boldsymbol{\alpha}^{\mathrm{A}}\right|\left(1-3 \cos ^{2} \phi_{\mathrm{A}}\right)\left(\cos \Theta-3 \cos \phi_{\mathrm{A}} \cos \phi_{\mathrm{B}}\right)
$$

respectively. Both (3.10) and (3.11) share the characteristics of comprising two groups of factors, the first representing the molecular electronic properties and the second the orientational dependence.

Taking the above results and employing the golden rule, expression (2.5), yields graphable results for the rate, $\Gamma(\Theta)$, of three-body energy pooling within such chromophoric arrays. On choosing $\Theta$, surfaces may then be plotted to illustrate the rate dependence on $\phi_{\mathrm{A}}$ and $\phi_{\mathrm{B}}$. The geometrically interesting situations of $\Theta=0, \pi / 2$ and $\pi$ are shown on the surfaces contained in Figs. 5 and 6. These 3D plots clearly exhibit the angular orientations most, and least, amenable to three-centre energy pooling. When the transition moments are orthogonal to the intermolecular vectors (i.e. if $\phi_{\mathrm{A}}$ and/or $\phi_{\mathrm{B}}=\pi / 2$ or $3 \pi / 2$ ) low transfer rates are predicted with $\Theta$ having a negligible effect. For the rate maxima, however, a more prominent dependence on $\Theta$ is observed. When $\Theta=0$, i.e. where all transition moments are coplanar, it can be seen that the rate maxima on both the surface for $\mathrm{ABA}^{\prime}$ and also that for $\mathrm{AA}^{\prime} \mathrm{B}$ occur at the $\left(\phi_{\mathrm{A}}, \phi_{\mathrm{B}}\right)$ coordinates $(0, \pi),(\pi, 0),(\pi, 2 \pi)$ and $(2 \pi, \pi)$. However if $\Theta=\pi / 2$ (transition moment planes orthogonal) or $\Theta=\pi$ (again coplanar), rate maxima occur at coordinates $(0$, $0),(0,2 \pi),(\pi, \pi),(2 \pi, 0)$ and $(2 \pi, 2 \pi)$, again for both $\mathrm{ABA}^{\prime}$ and $\mathrm{AA}^{\prime} \mathrm{B}$.

\section{Discussion}

In photoactive systems designed to exploit three-centre energy pooling, it will be important to optimise photophysical efficiency by having proper regard to the chromophore layout. In this context it is worth considering a number of physically important implications which can be drawn out of the results given in Section 3. In so doing, it should also be emphasised again that the linear arrays which have been the focus of the detailed calculation, have been chosen primarily to illustrate the much more extensive possibilities for application of the general results of eqns. (2.8).

Consider first the geometric factors which control the relative contributions of the single cooperative and the two accretive mechanisms within each system, as given in eqns. (3.10) and (3.11) respectively. In an $\mathrm{ABA}^{\prime}$ configuration, represented by eqn. (3.8), the dominant method of transfer is cooperative pooling, signified by $C^{\text {coop }}$-since the premultiplier of $\frac{1}{4}$ reduces the effectiveness of the accretive contribution $C^{\text {acc }}$ Conversely, in an $\mathrm{AA}^{\prime} \mathrm{B}$ chromophore arrangement, described by eqn. (3.9), the 36 -fold comparative increase in the accretive contributions serves to reduce the potency of the cooperative pathway. The exchange in dominance of the two pooling mechanisms stems purely from the difference in the relative interchromophore displacements in the $\mathrm{ABA}^{\prime}$ and $\mathrm{AA}^{\prime} \mathrm{B}$ arrangements.

Secondly, alongside the angular and positional considerations, the relative magnitudes of the molecular components of eqns. (3.10) and (3.11) also exert a degree of control over the rate and method of three-centre energy pooling. Specifically, with transition moments for all chromophores roughly equivalent, the main director of mechanistic influence is the magnitude of the generalised polarisability (2.9). The geometric predilection for one pooling mechanism over another can be

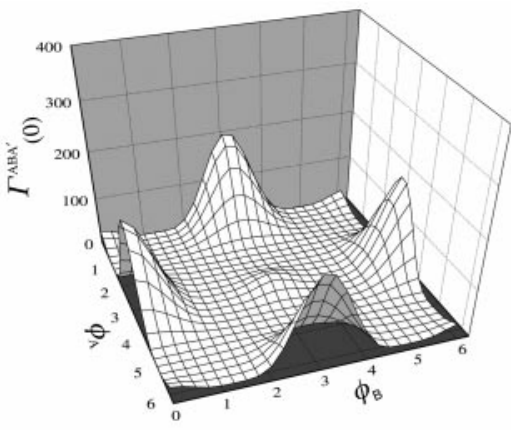

(a)

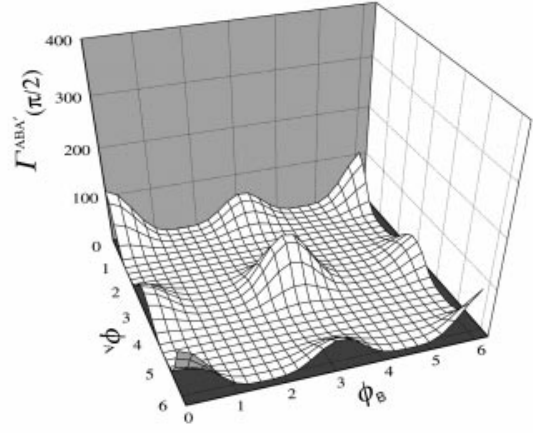

(b)

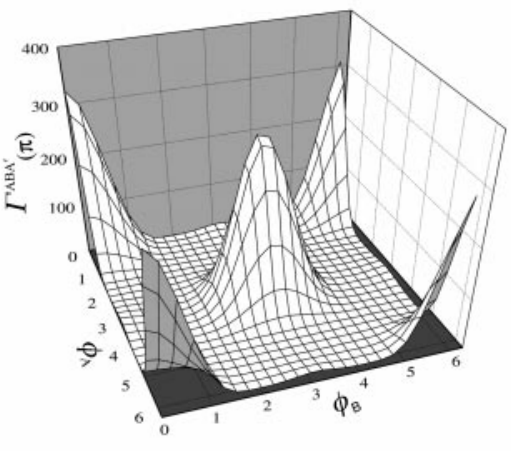

(c)

Fig. $53 \mathrm{D}$ plots illustrating how the rate of trichromophore energy pooling for a molecular architecture favouring cooperative transfer, $\Gamma^{\mathrm{ABA}}(\Theta)$, varies with angles $\phi_{\mathrm{A}}$ and $\phi_{\mathrm{B}}$ at fixed $\Theta:$ (a) $\Theta=0$, (b) $\Theta=\pi / 2$, (c) $\Theta=\pi$.

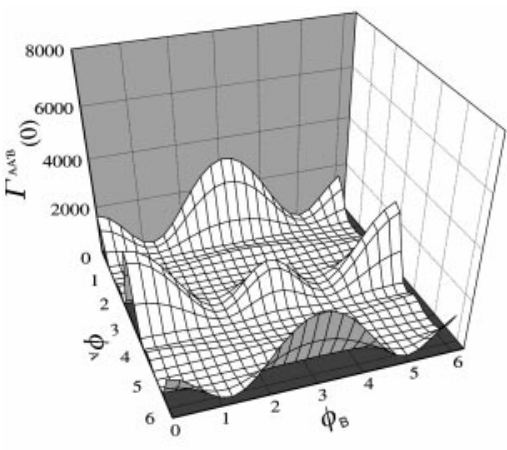

(a)

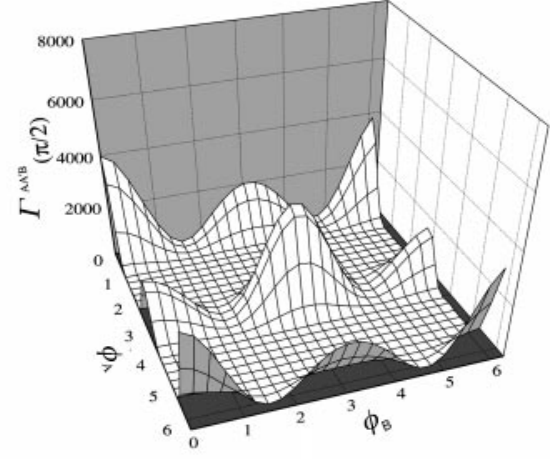

(b)

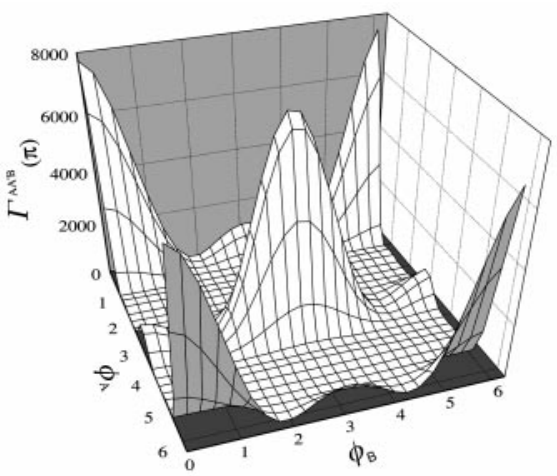

(c)

Fig. $63 \mathrm{D}$ plots illustrating how the rate of trichromophore energy pooling for a molecular architecture favouring accretive transfer, $\Gamma^{\mathrm{AA}}{ }^{\prime} \mathrm{B}(\Theta)$, varies with angles $\phi_{\mathrm{A}}$ and $\phi_{\mathrm{B}}$ at fixed $\Theta$ : (a) $\Theta=0$, (b) $\Theta=\pi / 2$, (c) $\Theta=\pi$. 


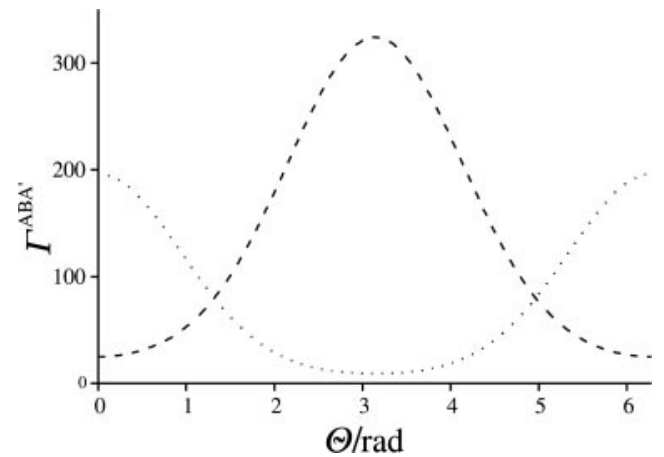

Fig. 7 Plot showing how rate maxima in ABA' configurations vary with $\Theta$ the $\left(\phi_{\mathrm{A}}, \phi_{\mathrm{B}}\right)$ angular dispositions $\{(0, \pi),(\pi, 0),(\pi, 2 \pi),(2 \pi, \pi)\}$ (dotted line) and $\{(0,0),(0,2 \pi),(\pi, \pi),(2 \pi, 0),(2 \pi, 2 \pi)\}$ (dashed line).

compensated by employing chromophores with suitably large polarisabilities. The import of $C^{\text {coop }}$ is enhanced by a large acceptor polarisability whereas $C^{\text {acc }}$ benefits from a sizeable donor polarisability. The implication here is that, for example, even with an architecture biased toward the cooperative pathway (such as $\mathrm{ABA}^{\prime}$ ), the ulterior accretive mechanisms can still play a significant role with a suitable choice of donor chromophore.

By identifying strategies for maximising the rates of energy pooling in arrays with linear trichromophore architecture, other broad implications can be identified for real systems. Figs. 5 and 6 demonstrated two sets of angular dispositions capable of yielding optimum pooling rates, for both the $\mathrm{ABA}^{\prime}$ and $\mathrm{AA}^{\prime} \mathrm{B}$ configurations. The difference between the corresponding optimal coordinate pairs, $\left(\phi_{\mathrm{A}}, \phi_{\mathrm{B}}\right)$, results from different values of the $\left(\cos \phi_{\mathrm{A}} \cos \phi_{\mathrm{B}}\right)$ feature of both (3.10) and (3.11). Specifically the first set, $(0, \pi),(\pi, 0),(\pi, 2 \pi)$ and $(2 \pi, \pi)$, yields the value of -1 and the second, $(0,0),(0,2 \pi),(\pi, \pi),(2 \pi, 0)$ and $(2 \pi, 2 \pi)$, yields +1 . Using the $\mathrm{ABA}^{\prime}$ system as an example, the first set of angular configurations, assuming molecular contributions to be normalised to unity, provides the following expression for the rate maxima in terms of the angle between transition moment planes:

$$
\Gamma_{\max }^{\mathrm{ABA}^{\prime}} \propto\left\{(\cos \Theta+3)^{2}-\frac{1}{2}(\cos \Theta+3)\right\}^{2} .
$$

Similarly the second set of points leads to

$$
\Gamma_{\max }^{\mathrm{ABA}^{\prime}} \propto\left\{(\cos \Theta-3)^{2}-\frac{1}{2}(\cos \Theta-3)\right\}^{2} .
$$

Fig. 7 shows a plot of the energy pooling maxima and serves to illustrate the most favourable molecular geometries. The overall maximum transfer rate is obtainable when $\Theta=\pi$. The maximum achievable transfer has its lowest ceiling at the points where the two functions intersect, revealing the conditions least well-suited to transfer. Equating (4.1) to (4.2) estab-

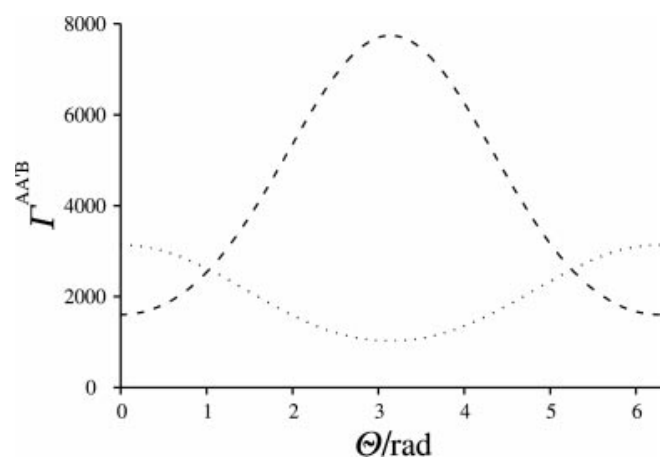

Fig. 8 Plot showing how rate maxima in AA'B architectures vary with $\Theta$ for $\left(\phi_{\mathrm{A}}, \phi_{\mathrm{B}}\right)=\{(0, \pi),(\pi, 0),(\pi, 2 \pi),(2 \pi, \pi)\}$ (dotted line) and $\{(0,0),(0,2 \pi),(\pi, \pi),(2 \pi, 0),(2 \pi, 2 \pi)\}$ (dashed line). lishes this as the case where $\Theta=\cos ^{-1}\left(\frac{1}{4}\right)$. Similarly for the AA $^{\prime}$ B configuration, represented by Fig. 8, the crossing point is where $\Theta=\cos ^{-1}[3(3-2 \sqrt{2})]$. In a conventional kappadependent transfer system ${ }^{53}$ the comparable geometry corresponds to $\Theta=\pi / 2$.

The molecular architectures discussed in Section 3 are the simplest representations of trichromophore arrays. In the literature, photoactive transfer systems abound, with more being reported on a regular basis. The examples above only begin to suggest methods of investigation into the performance and geometric optimisation of such arrays at high levels of illumination. For example, there is obviously rich scope for further investigation of the internal angle $\Phi$ whose primary effects have been presented elsewhere. ${ }^{38}$ It is hoped that the fully general results and methods reported here will serve to encourage and expedite the further analysis of such issues.

\section{Acknowledgement}

RDJ would like to express his thanks to the Engineering and Physical Sciences Research Council for remuneration.

\section{References}

1 A. A. Demidov and A. Y. Borisov, Biophys. J., 1993, 64, 1375.

2 R. Van Grondelle, J. P. Dekker, T. Gillbro and V. Sundström, Biochim. Biophys. Acta, 1994, 1184, 1.

3 A. A. Demidov and D. L. Andrews, Photochem. Photobiol., 1996 63, 39.

4 K. Sienicki, J. Chem. Phys., 1991, 94, 617.

5 E. Vuorrimaa, H. Lemmetyinen, P. Ballet, M. Van der Auweraer and F. C. De Schryver, Langmuir, 1997, 13, 3009.

6 H.-L. Wang, D. W. McBranch, V. I. Klimov, R. Hegleson and F. Wudl, Chem. Phys. Lett., 1999, 315, 173.

7 S. Woutersen and H. J. Bakker, Nature, 1999, 402, 507.

8 L. Stryer, Annu. Rev. Biochem., 1978, 47, 819.

9 P. R. Selvin, in Methods in Enzymology, vol. 246, ed. K. Sauer, Academic Press, Orlando, FL, 1995, p. 300.

10 P. Allcock, R. D. Jenkins and D. L. Andrews, Chem. Phys. Lett., 1999, 301, 228.

11 P. Allcock, R. D. Jenkins and D. L. Andrews, Phys. Rev. A, 2000 61, 3812.

12 T. Förster, Ann. Phys., 1948, $2,55$.

13 J. S. Avery, Proc. Phys. Soc. (London), 1966, 88, 1.

14 D. L. Andrews, Chem. Phys., 1989, 135, 195

15 H. Kuhn, J. Chem. Phys., 1970, 53, 101

16 G. Juzeliūnas and D. L. Andrews, in Resonance Energy Transfer, ed. D. L. Andrews and A. A. Demidov, John Wiley and Sons, Chichester, 1999, p. 65. (and references therein)

17 B. Valeur, in Fluorescent Biomolecules: Methodologies and Applications, ed. D. M. Jameson and G. D. Reinhardt, Plenum Press, New York, 1989, p. 296.

18 S. A. Latt, H. T. Cheung and E. R. Blout, J. Am. Chem. Soc., $1965,87,995$

19 Y. Ren, Z. Wang, H. Zhu, S. J. Weinberger and W. G. McGimpsey, J. Am. Chem. Soc., 1995, 117, 4367

20 B. Valeur, J. Mugnier, J. Pouget, J. Bourson and F. Santi, J. Phys. Chem., 1989, 93, 6073.

21 M. Kaschke, N. P. Ernsting, B. Valeur and J. Bourson, J. Phys Chem., 1990, 94, 5757.

22 D. Vyprachtický, V. Pokorná and F. Mikeš, Macromol. Chem. Phys., 1995, 196, 659.

23 M. N. Berberan-Santos and B. Valeur, J. Chem. Phys., 1991, 95, 8048.

24 L. B.-Å. Johansson and J. Karolin, Pure Appl. Chem., 1997, 69, 761.

25 P. G. Van Patten, A. P. Shreve, J. S. Lindsey and R. J. Donohoe, J. Phys. Chem. B, 1998, 102, 4209.

26 D. Kuciauskas, P. A. Liddell, S. Lin, T. E. Johnson, S. J. Weghorn, J. S. Lindsey, A. L. Moore, T. A. Moore and D. Gust, J. Am. Chem. Soc., 1999, 121, 8604.

27 M. N. Berberan-Santos, P. Choppinet, A. Fedorov, L. Jullien, B. Valeur, J. Am. Chem. Soc., 1999, 121, 2526 and references therein.

28 J.-S. Hsiao, B. P. Kreuger, R. W. Wagner, T. E. Johnson, J. K. Delaney, D. C. Mauzerall, G. R. Fleming, J. S. Lindsey, D. F. Bocian and R. J. Donohoe, J. Am. Chem. Soc., 1996, 118, 11181.

29 P. Brodard, S. Matzinger, E. Vauthey, O. Mongin, C. Papamicaël and A. Gossauer, J. Phys. Chem., A, 1999, 103, 5858. 
30 C. Devadoss, P. Bharathi and J. S. Moore, J. Am. Chem. Soc., 1996, 118, 9635

31 S. Tretiak, V. Chernyak and S. Mukamel, J. Phys. Chem. B, 1998, 102, 3310 .

32 D. L. Andrews and P. Allcock, Chem. Soc. Rev., 1995, 24, 259.

33 Resonance Energy Transfer, ed. D. L. Andrews and A. A. Demidov, John Wiley and Sons, Chichester, 1999.

34 K. Kilså, J. Kajanus, J. Mårtensson and B. Albinsson, J. Phys. Chem. B, 1999, 103, 7329.

35 D. P. Craig and T. Thirunamachandran, Chem. Phys., 1989, 135, 37.

36 G. D. Scholes, K. P. Ghiggino, A. M. Oliver and M. N. PaddonRow, J. Phys. Chem., 1993, 97, 11871.

37 K. P. Ghiggino, E. K. L. Yeow, D. J. Haines, G. D. Scholes and T. A Smith, J. Photochem. Photobiol. A: Chem., 1996, 102, 81.

38 R. D. Jenkins and D. L. Andrews, J. Phys. Chem. A, 1998, 102 10834.

39 R. D. Jenkins and D. L Andrews, Chem. Phys Lett., 1999, 301, 235.

40 R. D. Jenkins and D. L. Andrews, work in progress.

41 P. Allcock and D. L. Andrews, J. Chem. Phys., 1998, 108, 3089.

42 P. Allcock and D. L. Andrews, in OSA Trends in Optics and Photonics: Biomedical Optical Spectroscopy and Diagnostic/ Therapeutic Laser Applications, vol. 22, ed. E. M. SevickMuraca, J. A. Izatt and M. N. Ediger, Optical Society of America, Washington, DC, 1994, p. 84.
43 M. Chua and P. A. Tanner, J. Lumin., 1996, 66\&67, 203.

44 D.-L. Jiang and T Aida, Nature, 1997, 388, 454.

45 F. Barigelletti and L. Flamigni, Chem. Soc. Rev., 2000, 29, 1.

46 D. P. Craig and T. Thirunamachandran, Molecular Quantum Electrodynamics, Dover, New York, 1998.

47 J. Knoester and S. Mukamel, Phys. Rev. A, 1989, 40, 7065.

48 G. Juzeliūnas, Chem. Phys., 1995, 198, 145.

49 G. Juzeliūnas, Phys. Rev. A, 1996, 53, 3543

50 D. L. Andrews, S. Naguleswaran and G. E. Stedman, Phys. Rev. $A, \mathbf{1 9 9 6 , 5 7 , 4 9 2 5 .}$

51 L. C. Dávila Romero, S. Nagulewaran, G. E. Stedman and D. L. Andrews, Nonlinear Opt., in press.

52 D. L. Andrews, J. Raman Spectrosc., in press.

53 J. Baumann and M. D. Fayer, J. Chem. Phys., 1986, 85, 4087.

54 B. W. Van der Meer, G. Coker III and S.-Y. Chen, Resonance Energy Transfer Theory and Data, VCH, New York, 1994.

55 B. W. Van der Meer, in Resonance Energy Transfer, ed. D. L. Andrews and A. A. Demidov, John Wiley and Sons, Chichester, 1999, p. 151.

56 P. O'Hara, S. M. Yeh, C. F. Mears and R. Bersohn, Biochemistry, $1981,20,4704$

57 Z. Hillel and C.-W. Wu, Biochemistry, 1976, 15, 2105.

58 J. V. Mersol, H. Wang and D. G. Steel, Biophys. J., 1992, 61, 1647.

59 B. Nickel, Chem. Phys., 1995, 198, 353. 